\title{
A experiência de monitoramento do Programa Nacional de Capacitação dos Trabalhadores do SUAS (CapacitaSUAS)*
}

Patrícia Augusta Ferreira Vilas Boas ${ }^{1}$ Marcílio Marquesini Ferrari ${ }^{2}$

Maria Cristina Abreu Martins de Lima ${ }^{3}$ Michelle Conceição Stephanou ${ }^{4}$ Renato Silveira Souza Monteiro ${ }^{5}$

Davi Lopes Carvalho ${ }^{6}$

* Este artigo foi originalmente apresentado no VI Seminário RBMA (2014). Para esta publicação, seus dados foram atualizados.

1 Mestra em Educação. Atualmente é Diretora do Departamento de Formação e Disseminação (DFD) da Secretaria de Avaliação e Gestão da Informação (SAGI) do Ministério do Desenvolvimento Social e Combate à Fome (MDS).

2 Mestre em Ciência Política. É Coordenador-geral de formação de agentes públicos e sociais do DFD/SAGI/MDS.

3 Mestra em Políticas Sociais. Coordenadora-geral de disseminação do DFD/SAGI/MDS.

4 Mestra em Ciências Sociais. Coordenadora de disseminação do DFD/SAGI/MDS.

5 Especialista em produção de conteúdos e roteiros para a produção de materiais de cursos em EAD. Consultor Unesco Projeto 914BRZ3002.

6 Mestre em Informática Aplicada. Coordenador-geral de informações estratégicas do Departamento de Gestão da Informação - DGI/SAGI/MDS. 


\section{Resumo}

O Programa nacional de Capacitação de TraBALHADORES DO SISTEMA ÚNICO DE ASSISTÊNCIA SOCIAL (CAPACITASUAS), PUBLICADO EM MARÇO DE 2012 E COM A PRIMEIRA OFERTA REALIZADA EM AGOSTO DE 2013, CERTIFICOU, ATÉ OUTUBRO DE 2015, UM TOTAL DE 19.156 TRABALHADORES DO SUAS, SENDO OUE 15.130 ESTAVAM EM PROCESSO DE CAPACITAÇÃO ATÉ AOUULA DATA. A AROQUITETURA DE MONITORAMENTO E A AVALIAÇÃo do Programa CAPACITASUAS CONSIDERAM SUAS CARACTERÍSTICAS DE EXECUÇÃO DESCENTRALIZADA EM TODO O TERRITÓRIO NACIONAL E A PRESENÇA DE ATORES VARIADOS COM DIFERENTES RESPONSABILIDADES NO PROCESSO. ESTE ARTIGO APRESENTA A METODOLOGIA E OS RESULTADOS DE MONITORAMENTO DO CAPACITASUAS NOS ESFORÇOS OUE SÃo COORDENADOS PELO DEPARTAMENTO DE FORMAÇÃO E DISSEMINAÇÃo (DFD) dA SECRETARIA dE AvALIAÇÃO E GESTÃO DA INFORMAÇÃO (SAGI) DO MINISTÉrio do DESENVOLVIMENTO SOCIAL E COMBATE À FoMe (MDS).

\section{Abstract}

The Programa nacional de Capacitação de TraBALHADORES DO SISTEMA ÚNICO DE ASSISTÊNCIA SOcial (Capacitasuas) [National Training Program FOR SOCIAL WORKERS FROM THE UNIFIED SOCIAL AsSistanCE SYSTEM], LAUNCHED IN MARCH 2012 AND FIRST OFFERED IN AUGUST 2013, CERTIFIED 19,156 WORKERS UNTIL OCTOBER 2015, WHEN THERE WERE ALSO 14,681 WORKERS IN THE TRAINING PROCESS. THE MONITORING AND EVALUATING ARCHITECTURE OF THE CAPACITASUAS PROGRAM CONSIDERS ITS DECENTRALIZED IMPLEMENTATION CHARACTERISTICS FOR THE ENTIRE NATIONAL TERRITORY AND THE PRESENCE OF DIFFERENT ACTORS WITH ASSORTED RESPONSIBILITIES IN THE PROCESS. THIS ARTICLE PRESENTS THE METHODOLOGY AND RESULTS IN THE MONITORING OF CAPACITASUAS IN THE EFFORTS THAT ARE COORDINATED BY THE DEPARTAMENTO DE FORMAÇÃO E DISSEMINAÇÃO (DFD) [TRAINING AND DISSEMINATION DEPARTMENT] of the SeCREtaria de AVALIAÇÃo E GEStÃo da INFORMAÇÃO (SAGl) [SECRETARIAT FOR EVALUATION AND INFORMATION MANAGEMENT] OF THE MINISTÉRIO do Desenvolvimento Social e Combate à Fome (MDS) [MINISTRY OF SOCIAL DEVELOPMENT AND FIGHT AGAINST HUNGER].

\section{PALAVRAS-CHAVE:}

Monitoramento; Capacitação; Trabalho social.

Revista Brasileira de Monitoramento e Avaliação | Número 9 | Janeiro-Junho de 2015

A experiência de monitoramento do Programa Nacional de Capacitação dos Trabalhadores do SUAS (CapacitaSUAS) 


\section{Introdução}

Organizada desde 2005 por meio do Sistema Único de Assistência Social (SUAS), a política pública de assistência social tem buscado, progressivamente, a ampliação de seu papel no âmbito da proteção social e da melhoria das condições de vida da população, conforme preconizado pela Constituição Federal de 1988. Após período recente de investimentos na assistência social no Brasil, com o aumento do número de equipamentos públicos, recursos humanos envolvidos, benefícios e serviços disponibilizados à população, as atenções se voltam para as necessidades de capacitação dos trabalhadores, a fim de aprimorar os resultados.

O SUAS exige um novo perfil de trabalhadores e de conselheiros, que devem romper com as práticas assistencialistas, clientelistas e preconceituosas historicamente presentes na política de assistência social. No âmbito do Distrito Federal, dos estados e dos municípios, um total de 270.571 trabalhadores encontram-se alocados nas unidades e nos equipamentos públicos vinculados ao SUAS, segundo o Censo SUAS 20147, dos quais 42.711 de nível fundamental, 131.064 de nível médio e 96.796 de nível superior.

Esses trabalhadores desenvolvem suas atribuições profissionais em uma área cujos avanços normativos e configurações organizacionais, de serviços e de gestão procuram instituir e colocar em prática um conjunto de ações finalísticas (serviços, benefícios, programas e projetos) e um modelo de gestão e financiamento. Ao atenderem ao comando constitucional que dotou a assistência social do status de direito, tais ações representam uma ruptura paradigmática com uma longa história em que o Estado atuava apenas no fomento de ações da chamada sociedade civil. Estas se apresentavam de forma fragmentada, descontínua, destituída de financiamento regular e orientadas pela lógica da caridade e da benemerência.

Nesse contexto, e sobretudo em resposta às deliberações das diversas conferências nacionais de assistência social, foi instituído o Programa Nacional de Capacitação do SUAS (CapacitaSUAS) pela Resolução CNAS nº 8, de 2012, que objetiva aprimorar as capacidades/competências de gestão, de provimento de serviços e benefícios e de controle social dos trabalhadores e conselheiros do SUAS por meio do apoio aos estados e ao Distrito Federal na execução dos planos estatuais de capacitação do SUAS.

Dessa forma, este artigo tem o escopo de apresentar a metodologia e os resultados do monitoramento do CapacitaSUAS nos esforços que são coordenados pelo Departamento de Formação e Disseminação (DFD) da Secretaria de Avaliação e Gestão da Informação (SAGI) do Ministério do Desenvolvimento Social e Combate à Fome (MDS). 


\section{O DESENHO DO PROGRAMA CAPACITASUAS}

O desenho do CapacitaSUAS prevê que somente Instituições de Ensino Superior (IES), Escolas de Governo e Institutos Federais de Educação credenciados à Rede Nacional de Capacitação e Educação Permanente do SUAS (Renep-SUAS) possam ser contratados para a capacitação dos trabalhadores no âmbito do programa. Atualmente, estão credenciadas à Renep-SUAS 121 IES, distribuídas em todo o território nacional, exceto nos estados do Acre e do Amapá.

O financiamento para o CapacitaSUAS conta com a transferência direta de recursos do governo federal por meio do Fundo Nacional de Assistência Social (FNAS) para os fundos estaduais de assistência social (FEAS), a partir de critérios técnicos estabelecidos no termo de aceite assinado pelos governos estaduais e federal, incluindo a pactuação de metas. 0 termo de aceite é o instrumento específico pelo qual os governos dos estados se comprometem a executar as metas de capacitação no âmbito do programa. Para a adesão ao CapacitaSUAS, todo estado deverá assinar voluntariamente o termo de aceite.

A estrutura institucional de coordenação federativa do programa compreende uma equipe de coordenação nacional, compartilhada entre a SAGI e a Secretaria Nacional de Assistência Social (SNAS), e equipes de coordenação nos estados e no Distrito Federal (DF), a cargo das respectivas secretarias estaduais de assistência social. É importante destacar que as equipes do Departamento de Gestão do SUAS (DGSUAS/SNAS) têm apoiado de forma decisiva a implementação das atividades de monitoramento do CapacitaSUAS.

Todo o processo de pactuação entre os entes federativos é submetido à Comissão Intergestores Tripartite (CIT), às comissões intergestores bipartite (CIBs), ao Conselho Nacional de Assistência Social (CNAS) e ao Conselho Estadual de Assistência Social (CEAS). Essa estrutura dialoga e se submete à dinâmica própria do processo de pactuação e aos instrumentos regulares de planejamento e controle social do SUAS em cada nível federativo, conforme fluxo apresentado a seguir.

7 O Censo SUAS é um processo de monitoramento que, desde 2007, coleta dados sobre o SUAS por meio de um formulário eletrônico preenchido pelas secretarias e conselhos de assistência social dos estados e municípios. A base de dados do Censo SUAS 2014 está disponível em: http://aplicacoes.mds.gov.br/sagirmps/ferramentas/docs/Censo\%20SUAS\%202014_Versao_ atualizada.pdf

Revista Brasileira de Monitoramento e Avaliação | Número 9 | Janeiro-Junho de 2015 


\section{- FIGURA 1: FLUXOS DE COMUNICAÇÃO ENTRE SUJEITOS INSTITUCIONAIS ENVOLVIDOS NO PLANEJAMENTO DAS AÇÕES DO CAPACITASUAS}

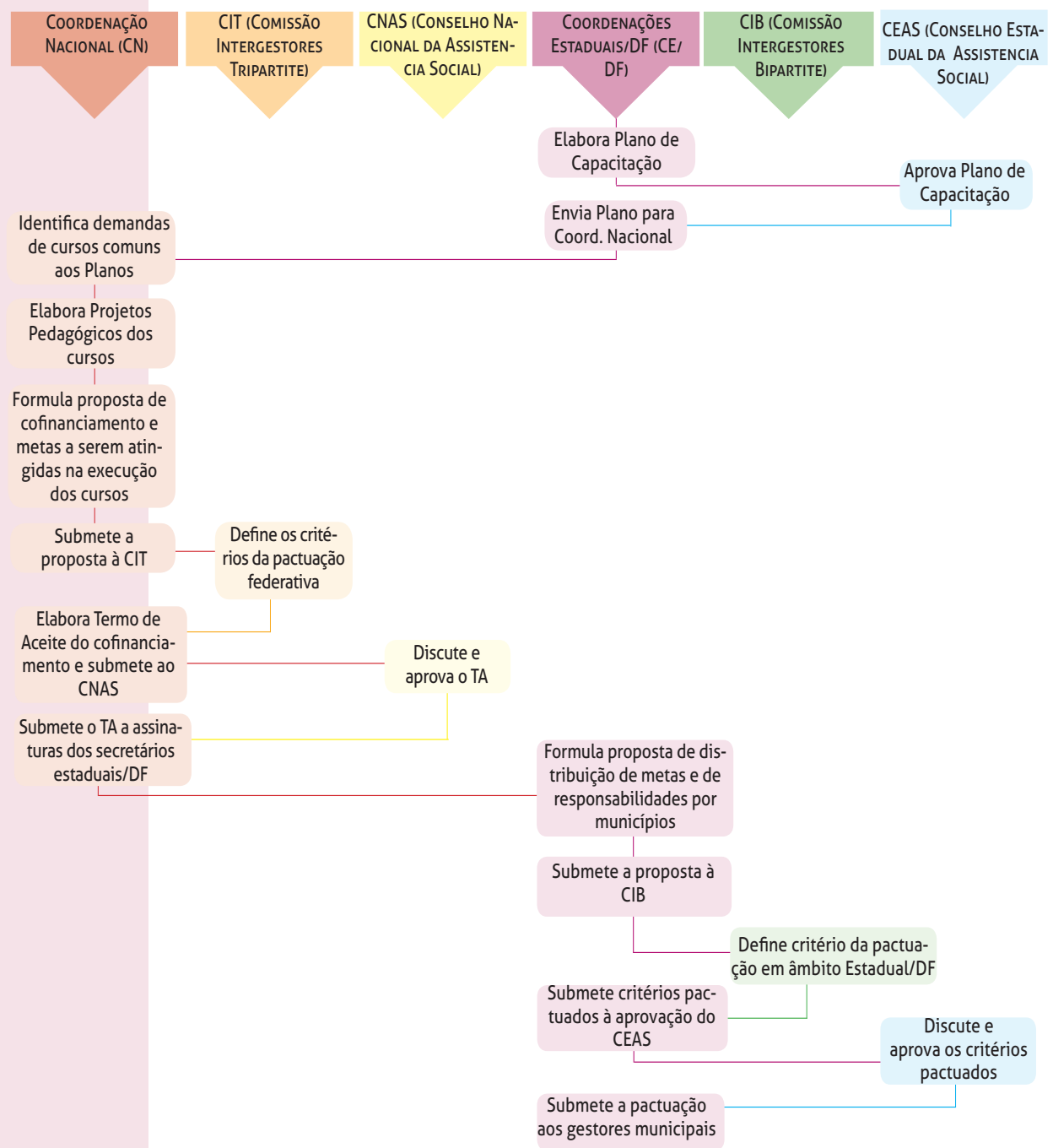

Fonte: Elaboração dos autores.

Após cumprir o percurso de pactuação ordinário da política, foram disponibilizados três cursos para a primeira fase do programa (Tabela 1). É importante apontar que as soluções de capacitação (desenhadas em nível federal e ofertadas aos estados para que estabeleçam contrato com instituições da Renep-SUAS) são elaboradas com base nas necessidades indicadas nos planos estaduais de capacitação e podem ser adaptadas às realidades e às necessidades regionais na fase de execução ${ }^{8}$. 
TABELA 1: CURSOS OFERTADOS PELO PROGRAMA CAPACITASUAS 2013-2014

\section{CURSOS}

PÚBLICO

\section{OBJETIVO}

Introdução ao Provimento dos Serviços e Benefícios Socioassistenciais do SUAS e à Implementação de Ações do Brasil Sem Miséria (BSM)

Profissionais de nível superior que atuam na provisão dos serviços socioassistenciais no âmbito dos CRAS, CREAS, centros pops, serviços de acolhimento institucional e serviços volantes.
Realizar nivelamento dos profissionais de nível superior que atuam no provimento dos serviços socioassistenciais quanto aos conhecimentos, habilidades e atitudes necessários ao provimento dos serviços e benefícios socioassistenciais e à implementação das ações do BSM.
Atualização em Indicadores para Diagnóstico e Acompanhamento do SUAS e do Brasil Sem Miséria (BSM)
Gestores e técnicos de nível superior das secretarias de assistência social dos estados, do Distrito Federal e dos municípios e coordenadores de CRAS e CREAS.
Capacitar gestores e técnicos estaduais e municipais para realizar diagnósticos, levando em consideração o contexto socioeconômico e a dimensão da pobreza nos estados e municípios, de forma a subsidiar a elaboração dos planos municipais de assistência social, bem como o acompanhamento dos programas e ações do SUAS e BSM.
Atualização em Gestão Financeira e Orçamentária do SUAS
Técnicos de nível superior que atuam diretamente nas atividades de gestão dos fundos de assistência social.

\begin{abstract}
Fornecer aos trabalhadores que atuam diretamente nas atividades de gestão dos fundos de assistência social subsídios conceituais e tático-operativos atualizados para os processos e procedimentos relacionados ao sistema de planejamento orçamentário brasileiro e à gestão financeira e orçamentária do SUAS.
\end{abstract}

\section{Metodologia}

A estrutura organizacional do MDS conta com a SAGI, cujas principais atribuições estão relacionadas à construção da avaliação, do moni- toramento, da gestão da informação e da realização de capacitações e ações de formação vinculadas às políticas públicas de desenvolvimento social. Na SAGI há um acordo conceitual que define monitoramento como

8 Os projetos pedagógicos dos cursos CapacitaSUAS são elaborados na perspectiva da educação permanente, entendida como o processo de atualização e renovação contínua das práticas e atitudes profissionais das equipes de trabalho e diferentes agrupamentos, a partir da afirmação de valores e princípios e do contato com novos aportes teóricos, metodológicos, científicos e tecnológicos disponíveis (PNEP-SUAS, 2013).

Revista Brasileira de Monitoramento e Avaliação | Número 9 | Janeiro-Junho de 2015 
[...] acompanhamento contínuo, cotidiano, por parte de gestores e gerentes, do desenvolvimento dos programas e políticas em relação a seus objetivos e metas. É uma função inerente à gestão dos programas, devendo ser capaz de prover informações sobre o programa para seus gestores, permitindo a adoção de medidas corretivas para melhorar sua operacionalização (VAITSMAN; PAES-SOUSA; RODRIGUES, 2006).

Por um lado, as atividades de monitoramento ocorrem como um acompanhamento próprio da atividade de gestão. Nesse âmbito, as ações empreendidas pelo MDS para o monitoramento do programa podem ser sintetizadas dessa forma:

a. a) Acompanhamento sistemático da atual fase de execução em cada estado e registro nos Sistema de Gestão Estratégica da SAGI (SIGE) e Sistema de Monitoramento Acadêmico (SIMA). O SIGE é um sistema de monitoramento de programas e projetos desenvolvido pela SAGI por meio do Departamento de Gestão da Informação (DGI), que possibilita aos gestores nacionais do CapacitaSUAS o acompanhamento sistemático da execução em cada estado. As informações são alimentadas a partir das atualizações encaminhadas formalmente pelos estados ao ponto focal do DFD/SAGI, por e-mail ou telefone. O SIMA é um sistema desenvolvido e mantido pela SAGI capaz de receber as informações das capacitações executadas pelos estados e parceiros da Renep-SUAS, tratá-las e gerar relatórios. b. b) Assessoramento aos estados para a construção de Termos de Referência (TR) visando à contratação de IES que ofertarão os cursos.

c. c) Realização de oficinas de alinhamento pedagógico e de conteúdos com professores das IES contratadas e gestores estaduais do programa.

Por outro lado, o monitoramento também se refere a "processos presenciais" e checagens locais, que acabam se constituindo um tipo de pesquisa rápida, qualitativa, por meio da qual gestores, técnicos ou consultores podem verificar como a implementação está sendo realizada, se está atingindo seus objetivos e metas, além de verificar quais problemas estão interferindo nas ações, nos processos e na consecução dos objetivos previstos. Nessa perspectiva, as ações de monitoramento presencial do Programa CapacitaSUAS podem ser assim caracterizadas:

a. a) Construção de instrumento próprio de monitoramento para execução presencial dos cursos, contemplando basicamente dois itens: infraestrutura (condições físicas das salas de aula, acessibilidade, alimentação, alojamentos) e aspectos pedagógicos (respeito à matriz pedagógica acordada, dialogicidade, atividades pedagógicas desenvolvidas, favorecimento da aprendizagem significativa).

b. b) Visitas técnicas de monitoramento para aplicação do instrumento.

c. c) Elaboração e sistematização de relatórios de monitoramento. 
Dessa forma, os instrumentos de coleta e registro de dados utilizados pelo MDS para o monitoramento do programa são o SIGE, - SIMA, os relatórios de visitas técnicas de acompanhamento da execução dos cursos e as avaliações de reação dos cursistas, sistematizadas nos relatórios de execução do CapacitaSUAS produzidos pelos estados 9 .

As definições de planejamento do Programa CapacitaSUAS (quais cursos, quantas vagas etc.) ocorrem num ambiente que envolve multiplicidade de atores, como foi evidenciado na Figura 1. Por sua vez, a execução do programa ocorre de forma descentralizada, regida pelas relações contratuais entre o ente estadual e uma ou mais instituições de ensino que compõem a Renep-SUAS, a partir de orientações gerais e recursos repassados do nível federal.

Isso implica um grau de complexidade para as ações de monitoramento e aumenta a necessidade de clareza do programa, seus objetivos, prioridades e metas. Como alerta Vaz (2009), nessas condições é essencial saber o que monitorar, avançando ao máximo na percepção quanto aos resultados pretendidos. Dessa forma, assume-se o caráter processual da construção do modelo de monitoramento do CapacitaSUAS, no qual a etapa atual introduz estratégias de relacionamento com os diversos atores e de acompanhamento acerca dos insumos empregados, atividades desenvolvidas e produtos disponibilizados.

\section{Resultados e discussão}

Entre 2012 e 2013 foram transferidos R\$57.389.808,0010 aos estados para que cada um realizasse os procedimentos administrativos necessários para a efetivação de contratos com uma ou mais IES da Renep-SUAS. Todas as unidades federativas aderiram ao CapacitaSUAS. Em conjunto, os estados e - DF pactuaram a oferta de mais de $37 \mathrm{mil}$ vagas para os cursos disponibilizados para essa primeira fase (40 horas cada curso). Até outubro de 2015, o CapacitaSUAS certificou 19.156 trabalhadores e 15.130 vagas estavam contratadas pelos estados com alguma instituição da RENEP-SUAS. Esse conjunto de 34.286 trabalhadores representa $92 \%$ do total da meta estabelecida na primeira etapa de pactuação com os estados.

A Figura 2 mostra a evolução da implementação do CapacitaSUAS entre o segundo semestre de 2013 e o primeiro semestre de 2014.

9 A propósito, há que se registrar que também os estados e o DF possuem metodologias e instrumentos próprios de monitoramento do Programa CapacitaSUAS.

10 R\$ 27.375.433,00 foram repassados em 2012, e R\$ 27.014.375,00 em 2013. No final de 2014, foram empenhados mais R\$ 29.024.616,00; contudo, esses recursos de 2014 ainda não foram transferidos.

Revista Brasileira de Monitoramento e Avaliação | Número 9 | Janeiro-Junho de 2015 
- FIGURA 2: EVOLUÇÃO DA IMPLEMENTAÇÃO DO CAPACITASUAS

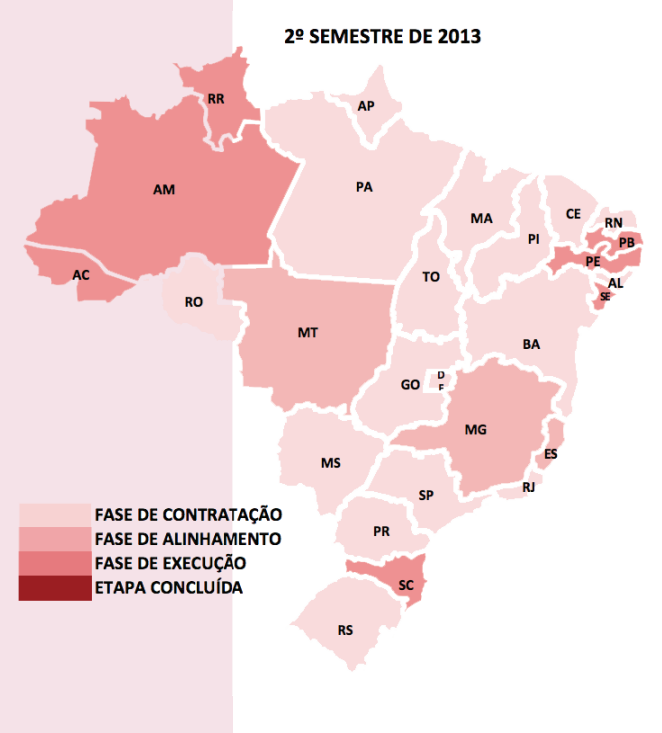

Fonte: Elaboração dos autores.

A execução dos primeiros cursos foi concluída em 15 estados: AC, AM, PA, RR, SC, PR, MG, DF, $E S, P B, S E, R N, D F, M S$ e MT. Oito estados se encontram em fase de conclusão: $\mathrm{BA}, \mathrm{MA}, \mathrm{PI}, \mathrm{CE}, \mathrm{PE}$, TO, RJ e RS. O estado de Goiás, após concluir o processo de contratação da instituição executora, deverá realizar os cursos ainda neste ano. Quatro estados ainda enfrentaram problemas de ordem administrativa e não conseguiram efetuar a contratação da IES executora: SP, AL, RO e AP.

No tocante aos registros efetuados no SIGE, sublinha-se que a partir de sua adoção há condições para que os gestores nacionais do programa acompanhem todo o histórico da evolução da execução contratual e os problemas enfrentados em todo o território nacional.

Foi possível perceber alguns entraves burocráticos. Por exemplo, ficou claro que há di-

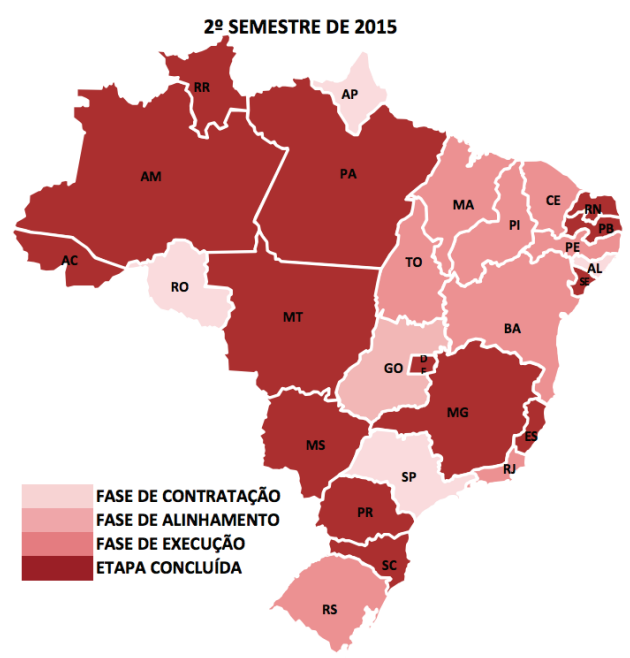

versas compreensões jurídicas por parte das procuradorias gerais dos estados a respeito do arranjo adotado no Programa CapacitaSUAS, fato que tem levado, em alguns casos, à demora na formalização de contratos administrativos ou congêneres. Há também dificuldades, por parte de algumas equipes estaduais, na elaboração dos Termos de Referência e na gestão de contratos administrativos estabelecidos com as IES da Renep/SUAS.

Para apoiar a superação dessas dificuldades houve disponibilização, por parte do DFD, de um termo de referência padrão para ser adotado/ adaptado por cada ente estadual, aliado a visitas técnicas realizadas com o objetivo de sanar dúvidas referentes ao documento. Essas ações contribuíram para acelerar e qualificar os processos formais de contratação de IES dos estados que receberam assessoria. As visitas técnicas aconte- 
ceram nos estados de Tocantins, Santa Catarina, Amazonas, Distrito Federal, Rio Grande do Norte, Piauí, Roraima, Ceará, Alagoas, Maranhão e Bahia. Percebeu-se que após a realização das visitas técnicas, alguns estados avançaram na elaboração e formalização de contratos administrativos, mas outros passaram por alterações em suas equipes, o que dificultou o início da execução do programa. Outros estados foram assessorados por telefone ou e-mail, tais como Paraná, Goiás, Espírito Santo, Rio Grande do Sul, Pará, São Paulo, Rio de Janeiro e Distrito Federal.

A Tabela 2 registra as diferentes formas de contratação estabelecidas no âmbito do CapacitaSUAS.

\section{- TABELA 2: FORMAS DE CONTRATAÇÃO ESTABELECIDAS PELOS ESTADOS PARA O CAPACITASUAS}

\begin{tabular}{|c|c|c|}
\hline UF & IES CONTRATADA & $\begin{array}{l}\text { FORMA DE CONTIRATA- } \\
\text { CÃO }\end{array}$ \\
\hline$A C$ & Centro Universitário do Sul de Minas (Unis) & Contrato por licitação \\
\hline AM & Universidade do Estado do Amazonas (UEA) & Convênio \\
\hline $\mathrm{BA}$ & Universidade Federal do Recôncavo da Bahia (UFRB) & Contrato por licitação \\
\hline DF & Centro Universitário do Sul de Minas (Unis) & Dispensa de licitação \\
\hline \multirow{2}{*}{ ES } & Universidade de Vila Velha (UVV) & \multirow{2}{*}{ Convênio } \\
\hline & Universidade Federal do Espírito Santo (UFES) & \\
\hline MT & Universidade Federal do Mato Grosso (UFMT) & Dispensa de licitação \\
\hline MS & Centro Universitário de Lins (Unilins) & Contrato por licitação \\
\hline MG & Universidade Federal de Minas Gerais (UFMG) & Contrato por licitação \\
\hline PA & Centro Universitário de Lins (Unilins) & Contrato por licitação \\
\hline PB & Universidade Federal da Paraíba (UFPB) & Convênio \\
\hline \multirow{5}{*}{ PR } & Universidade Estadual de Londrina (UEL) & \multirow{5}{*}{ Dispensa de licitação } \\
\hline & Universidade Estadual do Oeste do Paraná (Unioeste) & \\
\hline & Universidade Estadual de Ponta Grossa (UEPG) & \\
\hline & Universidade Estadual de Maringá (UEM) & \\
\hline & Universidade Estadual do Centro-Oeste do Paraná (Unicentro) & \\
\hline $\mathrm{PE}$ & Universidade Federal Rural de Pernambuco (UFRPE) & Contrato por licitação \\
\hline RJ & Universidade Federal do Rio de Janeiro (UFRJ) & Contrato por licitação \\
\hline RN & Universidade do Estado do Rio Grande do Norte (UERN) & Dispensa de licitação \\
\hline RR & Universidade Estadual de Roraima (UERR) & Convênio \\
\hline \multirow{2}{*}{ SC } & Universidade do Sul de Santa Catarina (Unisul) & \multirow{2}{*}{ Contrato por licitação } \\
\hline & Universidade Comunitária da Região de Chapecó (Unochapecó) & \\
\hline SE & Universidade Tiradentes (Unit) & Contrato por licitação \\
\hline
\end{tabular}

Revista Brasileira de Monitoramento e Avaliação | Número 9 | Janeiro-Junho de 2015 
Os registros efetuados no SIMA, por sua vez, permitem a extração de diversos relatórios para o acompanhamento do CapacitaSUAS. O sistema é alimentado pelas instituições ofertantes, que fazem a inserção dos dados de execução, registrando, inclusive, a avaliação de desempenho dos alunos; pelos estados, que inserem o planejamento da oferta, traduzido em número de vagas; e pela gestão federal do programa, que insere os cursos que são disponibilizados.

O SIMA está ativo, e o processo de preenchimento para o monitoramento do Programa CapacitaSUAS está em curso. No início da execução do programa e da utilização do sistema percebeu-se que o processo de pre- enchimento pelas instituições ofertantes se iniciou de forma lenta. Isso pode ser constatado pela baixa proporção de preenchimento (30\%) dos registros dos alunos certificados e em capacitação em outubro do ano de 2014. Diante desse quadro, o secretário da SAGI encaminhou ofício para as Instituições de Ensino e as equipes de gestão do trabalho dos estados solicitando o empenho no preenchimento do SIMA. Em outubro de 2015, a taxa de registro dos alunos certificados em capacitação atingiu 56\%. Esse processo de sensibilização e orientação das IES executoras para o preenchimento tempestivo do sistema tem sido intensificado pelo DFD/SAGI nas Oficinas de Alinhamento e nas visitas de monitoramento realizadas nos estados.

\section{TABELA 3: RELATÓRIOS CONSTANTES DO SIMA}
RELATÓRIOS
DESCRIÇÃO
Relatório de curso
Relatório de planeja- mento de oferta de curso
Relatório de acompanhamentos
Relatório de instituição
Relatório de gestores estaduais
Relatório de alunos registrados no CADSUAS
Relatório panorama nacional
Descreve cada curso promovido pelo MDS, seja ofertado diretamente, seja por parceiros. No re- gistro consta a ementa, a estrutura e a carga horária de cada curso. A estrutura de cada curso é pré-determinada, porém pode ser adaptada às necessidades locais, e é diferente para os cursos de formação e capacitação.
O planejamento da oferta do curso é cadastrado pelos coordenadores estaduais ou pelo próprio MDS, conforme o caso. Ele registra a oferta planejada de cada curso, com o número de vagas e data de início e fim das aulas. Também indica a instituição executora da oferta e o custo. Permite a visualização dos dados de oferta (sem a possibilidade de escolha de curso) por UF e o percentual de realização das capacitações inicialmente planejadas.
Descreve os principais dados cadastrais de uma instituição que pertença à Rede Nacional de Capacitação e Educação Permanente do SUAS (Renep-SUAS).
Registra os gestores estaduais de cada estado e do DF responsáveis por coordenar, no âmbito estadual e distrital, as ações de capacitação do CapacitaSUAS. Os dados dos gestores estaduais no Sima são preenchidos pela coordenação nacional e podem ser atualizados pelas próprias equipes de trabalho dos estados.
Permite a identificação de todos os cursistas com seus dados no CADSUAS, apontando os alunos que não fazem parte desse banco de dados.
Indica o consolidado de alunos certificados e em processo de capacitação por unidade da federa- ção. Permite a separação dos quantitativos de capacitados por programas e cursos. 
Em relação às ações presenciais de monitoramento do CapacitaSUAS, percebe-se, inicialmente, que a estratégia de realizá-las ao menos com as primeiras turmas de cada curso ofertado tem sido acertada. O acompanhamento da execução contou com a presença, em sala de aula, de técnicos do MDS, notadamente da SAGI (13) e da SNAS (04); consultores externos (07). Esses agentes públicos federais, em conjunto, realizaram observações em $7,6 \%$ do total de 850 turmas abertas ao longo da primeira etapa do CapacitaSUAS.

Considerando todos os registros até outubro de 2015, essas visitas geraram 65 relatórios de acompanhamento técnico da execução dos cursos da primeira etapa do programa. Desses, 39 referiam-se ao curso Introdução ao Provimento dos Serviços e Benefícios Socioassistenciais do SUAS e à Implementação de Ações do BSM (60\%); 11 relatórios referentes ao curso Atualização em Indicadores para Diagnóstico e Acompanhamento do SUAS e do BSM (17\%); e 15, relacionados ao curso Atualização em Gestão Financeira e Orçamentária do SUAS (23\%).

Em geral, os aspectos pedagógicos foram percebidos de forma mais positiva do que os de infraestrutura. Nesse campo, as salas de aula tiveram avaliação positiva em $83,1 \%$ dos casos; as salas para oficinas de aprendizagem, em $73,3 \%$; e a acessibilidade foi garantida em $81 \%$ das turmas acompanhadas. Os problemas apontados foram espaços inadequa- dos para acomodar confortavelmente a totalidade de cursistas nas turmas, com problemas de acústica e insuficiência de cadeiras e computadores; limpeza de banheiros; locais distantes para a realização dos cursos (aliado a problemas de transporte); falta de sinalização de locais importantes; e falta de coffee break.

No campo pedagógico, pode-se observar que a matriz sugerida para cada curso foi cumprida em $96,9 \%$ dos casos. No que se refere ao enfoque e aos objetivos instrucionais da matriz pedagógica, segundo observações dos técnicos e consultores do MDS, esses quesitos foram assegurados em 95,4\% das turmas acompanhadas. No tocante à carga horária estabelecida para cada módulo dos cursos, os relatórios mostraram o seu cumprimento em $90,6 \%$ das turmas.

Um aspecto positivo da metodologia adotada foi a ênfase na participação dos trabalhadores do SUAS. Dados dos relatórios revelam que $98,5 \%$ dos cursistas das turmas puderam participar de forma ativa e efetiva durante as aulas expositivas e dialogadas, e nas oficinas de aprendizagem. Essa metodologia, aplicada sob os princípios e diretrizes da Educação Permanente e da Andragogia, prioriza a consolidação do aprendizado teórico-conceitual e de sua aplicação à leitura da realidade, à avaliação de condutas alternativas e à resolução de problemas do cotidiano do trabalhador cursista. Em todas as turmas visitadas (100\%) constatou-se uma rica troca de experiência entre os participantes.

Revista Brasileira de Monitoramento e Avaliação | Número 9 | Janeiro-Junho de 2015 
Em alguns poucos casos, a metodologia de ensino foi questionada pelos cursistas que, como críticas principais, apontaram: discussões repetitivas; fuga do tema durante as discussões; leitura de slides pelo professor; abordagem excessivamente teórica em alguns momentos. Também foram feitas algumas observações sobre o material didático, indicando a necessidade de pequenas revisões/atualizações nos curso de introdução e de diagnóstico.

A presença da equipe estadual, da coordenação da IES e do MDS foi avaliada de forma positiva, na opinião dos observadores. Nos casos em que foi necessária uma intervenção para ajuste na execução, essa presença garantiu que as providências fossem tomadas em comum acordo e de forma tempestiva. Foi o caso, por exemplo, do uso inadequado do espaço da sala de aula para discussões trabalhistas, especialmente quanto aos salários, ou a capitalização política do evento de capacitação. Eventualmente, foram percebidas, por parte de alguns professores e cursistas, posições preconceituosas quanto ao público da assistência ou aos benefícios, programas, projetos e serviços da política de assistência social. É importante dizer que esses eventos puderam ser observados nos três cursos, o que ensejou o aprofundamento da discussão ética e política nas oficinas de alinhamento realizadas com os professores das IES executoras.

As Oficinas de Alinhamento têm por objetivo promover o nivelamento conceitual e de conteúdo entre as equipes de docentes das IES integrantes da Renep/SUAS. Até julho de
2015 foram realizadas nove oficinas, reunindo docentes, técnicos e gestores, todos articulados pelo órgão gestor do SUAS nos estados. As fichas de inscrição das oficinas, utilizadas a partir do quarto alinhamento, demonstram que, dentre os participantes, $87 \%$ têm mestrado ou doutorado, e somente 1\% é graduado, mas não possui nenhum título de pós-graduação. 54\% dos docentes são vinculados a Instituições de Ensino Públicas, e $46 \%$ a IES Privadas. Assistentes sociais são $67 \%$, e os demais têm formação em Psicologia, Sociologia, Pedagogia e Ciências Políticas, entre outros. Ao todo, já passaram pelas oficinas mais de 200 participantes de todas as regiões do país.

É interessante observar as informações que os monitoramentos presenciais trazem a respeito dos cursos. Em relação ao curso Introdução ao Provimento dos Serviços e Benefícios Socioassistenciais do SUAS, ressalta-se que a matriz pedagógica (objetivos/enfoque/ carga horária) ou foi elogiada quanto à sua pertinência ao cotidiano do trabalho, ou não foi criticada. $\mathrm{O}$ uso de recursos instrucionais variados e especialmente a oportunidade de troca de experiência em oficinas foram destacados positivamente. Porém, houve uma sugestão para que o Módulo I fosse reformulado para inserir uma atividade prática, com o intuito de avançar em dinamicidade, o que se percebeu não acontecer nas aulas expositivas/dialogadas previstas para esse módulo. Pode-se dizer que o curso contou com docentes avaliados positivamente pelos observadores. Eventuais desconhecimentos sobre conteúdos ou atualidades da área puderam ser superados com o estímulo à troca de ex- 
periências entre os aprendizes. Contudo, alguns docentes demonstraram desatualização normativa ou desconhecimento de temas da assistência. Nesses casos, foram realizadas reuniões com representantes do MDS, do estado e das IES para acordo sobre medida de substituição ou inserção de um docente adicional de forma rápida. Apenas nesses casos foi assinalado o cumprimento parcial da matriz pedagógica.

No curso Atualização em Indicadores para Diagnóstico e Acompanhamento do SUAS e do BSM, a disponibilidade de computadores para os alunos acompanharem o curso é fundamental. Esse aspecto de infraestrutura foi criticado pela insuficiência de equipamentos em algumas instituições de ensino. Foi assinalado o cumprimento satisfatório da matriz pedagógica e a existência de espaços para exercícios práticos. No entanto, houve sugestão para que as oficinas ganhassem mais aderência às necessidades do cotidiano por meio da apresentação de situações hipotéticas que estimulassem os aprendizes a buscar informações/soluções adequadas. Destaca-se que esse mesmo curso também vem sendo oferecido na modalidade de ensino a distância, por meio de uma parceria firmada entre a SAGI/ MDS e o Centro de Estudos Internacionais So- bre Governo (Cegov) da Universidade Federal do Rio Grande do Sul (UFRGS). Os professores das IES que ministram o curso no âmbito do CapacitaSUAS foram capacitados via EAD e tiveram acesso aos conteúdos (inclusive vídeos) para auxiliar a preparação de suas aulas.

O curso Atualização em Gestão Financeira e Orçamentária do SUAS teve a matriz pedagógica cumprida em todos os casos, tendo recebido adequação às especificidades locais. Os professores ganharam elogios, e as oficinas foram produtivas, criativas, participativas, segundo as avaliações de reação dos cursistas. No entanto, nas ofertas iniciais foi sugerido que o Módulo I se tornasse mais dinâmico, para a melhor compreensão dos conteúdos ${ }^{11}$.

Outra fonte importante de informações do monitoramento do CapacitaSUAS são os relatórios que os estados elaboram como parte do acordo firmado no termo de aceite. Por meio deles tornam-se conhecidas, pelo nível federal, as avaliações de reação dos alunos, além de outras informações sobre a execução do programa. As sínteses sobre as avaliações de reação encaminhadas por oito estados que já enviaram relatórios ( $P E, R N, M G, R R, A C, S E$ e PA) são coincidentes com os dados levantados no monitoramento presencial, apontando

11 É de sublinhar que os materiais didáticos de todos os cursos foram revisitados e aprimorados a partir das primeiras experiências de oferta do CapacitaSUAS no estados.

Revista Brasileira de Monitoramento e Avaliação | Número 9 | Janeiro-Junho de 2015 
satisfação com o curso e indicando que os aspectos pedagógicos foram ligeiramente melhor avaliados que os de infraestrutura ${ }^{12}$.

No que se refere aos desafios para o aprimoramento do programa, foi possível perceber que há poucos profissionais nas instituições da Renep-SUAS aptos a ministrar cursos voltados à temática do SUAS - ou seja, que estejam atualizados em relação à perspectiva da educação permanente e aos avanços da política e seus desafios -, embora estejam qualificados do ponto de vista acadêmico e com experiência de trabalho na área. Assim, reforça-se o papel das oficinas de alinhamento pedagógicas e de conteúdos como suporte importante para a superação desse problema.

Ressalta-se que essa é a primeira iniciativa de leitura dos dados do monitoramento em uma síntese única. Esse trabalho levou à percepção de necessidades de aperfeiçoamento, por exemplo, em relação ao instrumento de monitoramento utilizado nas visitas presenciais. Todavia, vale destacar que a adoção de um modelo único foi fundamental. Considera-se que a análise das mudanças necessárias, nesse caso, depende de um momento avaliativo a ser agendado com todo o grupo participante do processo. Percebe-se, além do mais, que é preciso obter com maior agilidade os dados de avaliação de reação para o monitoramento, e que o SIMA deve ser mais conhecido pelas instituições ofertantes. Cabe destacar, que o SIMA tem sido aprimorado constantemente a partir das contribuições oriundas de estados e instituições de ensino executoras. Outra agenda que se avizinha é a construção de pesquisas de avaliação de efeito do programa em todo o território nacional, que será realizada a partir de $2016^{13}$.

Os processos de monitoramento têm o objetivo principal de verificar o andamento do programa em relação aos seus objetivos e metas. Os dados apresentados mostram que o CapacitaSUAS está caminhando para o alcance das metas definidas pelos estados em seus planos estaduais. Por fim, a informação produzida pelo monitoramento permitiu a correção de rumos em diversas oportunidades, conforme relatado neste texto.

12 Considerando a relativa demora por parte dos estados em encaminhar formalmente os resultados das avaliações de reação, o DFD iniciou os procedimentos para a construção de um módulo de avaliação de reação no SIMA, em fase de testes, com vistas a disponibilizar para os alunos concluintes um link de preenchimento online da avaliação dos cursos. Outra novidade para a segunda etapa foi a criação de um instrumento para coletar as avaliações dos professores que participam do CapacitaSUAS a respeito do curso que ministraram e do programa.

13 Ainda na área de avaliação, é importante destacar que serão contratados dois consultores externos, que terão como meta a construção de indicadores qualitativos e quantitativos do Programa CapacitaSUAS, além de subsidiar a área técnica do DFD na construção de instrumentos para a avaliação de efeito do programa nos estados que já encerraram a primeira etapa. 


\section{Referências bibliográficas}

BRASIL. Ministério do Desenvolvimento Social e Combate à Fome. Caderno de Estudos Desenvolvimento Social em Debate, n. 22. Brasília, DF: MDS, 2015.

CONSELHO NACIONAL DE ASSISTÊNCIA SOCIAL (CNAS). Resolução CNAS nº 269, de 13 de dezembro de 2006. Institui a Norma Operacional Básica de Recursos Humanos do Sistema Único de Assistência Social - NOB-RH/SUAS. Brasília, DF, 2006.

Resolução CNAS nº 08, de 16 de março de 2012. Institui o Programa Nacional de Capacitação do SUAS - CapacitaSUAS e aprova os procedimentos e critérios para adesão dos Estados e do Distrito Federal ao cofinanciamento federal do Programa Nacional de Capacitação do SUAS - CapacitaSUAS. Brasília, DF, 2012.
Resolução CNAS n 04, de 13 de março de 2013. Institui a Política Nacional de Educação Permanente do SUAS. Brasília, DF, 2013.

VAITSMAN, J.; PAES-SOUSA, R.; RODRIGUES, R. W. S. $O$ sistema de avaliação e monitoramento das políticas e programas sociais: a experiência do Ministério do Desenvolvimento Social e Combate à Fome. Brasília, DF: Ministério do Desenvolvimento Social e Combate à Fome; UNESCO, 2006.

$V A Z$, J. C. 0 monitoramento no planejamento governamental em ambientes complexos: decisões e requisitos. Reflexões para a Ibero-América: planejamento estratégico. Brasília, DF: ENAP, 2009.

Revista Brasileira de Monitoramento e Avaliação | Número 9 | Janeiro-Junho de 2015 